\title{
I. Die Entstehung militärischer Strukturen in der DDR vor Gründung der NVA 1948-1956
}

Die Geschichte der DDR-Streitkräfte beginnt nicht erst mit der NVA-Gründung am 18. Januar 1956. Vielmehr hatte die SED bereits von 1948 bis 1952 durch den Aufbau kasernierter Polizeiverbände mit einer verdeckten Aufrüstung begonnen. Im Anschluß schuf sie zwischen 1952 und 1956 mit der KVP die militärische Vorläuferformation der NVA.

Im Mai 1948 erging die Weisung der Sowjetischen Militäradministration in Deutschland (SMAD) zum Aufbau kasernierter Polizeibereitschaften an die Deutsche Verwaltung des Innern ${ }^{1}$ (DVdI)2. Hierzu wurde im Juli 1948 die Hauptabteilung Grenzpolizei und Bereitschaften (HA GP/B) geschaffen, deren Leiter der spätere Chef Ausbildung im Ministerium für Nationale Verteidigung, Hermann Rentzsch, wurde. Die HA GP/B diente als Führungsorgan für die aufzustellenden kasernierten Polizeiverbände und die Grenzpolizei, die aus der Schutzpolizei herausgelöst worden war. Bis Oktober 1948 entstanden in allen Ländern der SBZ 40 Bereitschaften der Volkspolizei (VP) mit jeweils 250 Mann. Ende desselben Monats begannen die Bereitschaftsangehörigen mit ihrer Ausbildung, die zu diesem Zeitpunkt eindeutig auf eine militärische Befähigung ausgerichtet war. In einem nächsten Schritt wurden die VP-Bereitschaften und die Grenzpolizei auf Befehl des Präsidenten der DVdI, Kurt Fischer, bis Mitte November des Jahres aus den Polizeiformationen der Länder herausgelöst und der HA GP/B direkt unterstellt. Ende 1948 führte man zudem einheitliche Dienstbezeichnungen und Dienstgradabzeichen für die nunmehr insgesamt rund 9900 Bereitschafts- und 9300 Grenzpolizisten ein ${ }^{3}$.

Von großer Bedeutung für die weitere Entwicklung der bewaffneten Kräfte war zudem der Beschluß der SED-Führung vom 12. Juli 1948, auch in den Polizeiorganen SED-Parteistrukturen zu etablieren. Es war das Ziel der SED, von Beginn an auch in den VP-Bereitschaften ihre „führende Rolle“ durchzusetzen. $\mathrm{Zu}$ diesem

1 Die DVdI war schon im Juli 1946 auf Befehl der SMAD geschaffen worden. Ihr sollte die Leitung aller Institutionen der inneren Verwaltung sowie der öffentlichen Sicherheit und Ordnung obliegen. Ab 1948 fungierte sie als zentrales Führungsorgan aller Polizeikräfte und als Hilfsorgan für die effektivere Verwirklichung der sowjetischen Besatzungspolitik. Durch die Besetzung der Leitungspositionen der DVdI ausschließlich mit zuverlässigen Parteifunktionären konnte die SED ihren führenden Einfluß innerhalb des Polizei- und Sicherheitsapparates sicherstellen. Vgl. Eisert, Zu den Anfängen der Sicherheits- und Militärpolitik, S. $148 \mathrm{f}$.

2 Diese Entscheidung der UdSSR war vor allem ein Resultat der Londoner Konferenz vom Februar 1948. Dort hatten die Westmächte und die Benelux-Staaten den Aufbau eines föderativen Regierungssystems in den westdeutschen Besatzungszonen und deren Beteiligung am Marshallplan beschlossen. Die UdSSR entschied daraufhin ihrerseits, im Rahmen ihrer Doppelstrategie neben einer gesamtdeutsch ausgerichteten Politik den Aufbau staatlicher Strukturen in der SBZ zu verstärken. Dies betraf auch den Sicherheitsapparat als Stütze der neuen Gesellschaftsordnung. Vgl. Diedrich/Wenzke, Die getarnte Armee, S. $20 \mathrm{ff}$.

3 Vgl. Eisert, Zu den Anfängen der Sicherheits- und Militärpolitik, S. $166 \mathrm{ff}$. 
Zweck installierte die Partei bereits Ende 1948 einen speziellen Apparat für PolitKultur (PK). Allen Kommandeuren und Dienststellenleitern wurde als 1. Stellvertreter ein PK-Leiter zur Seite gestellt, der die konsequente Berücksichtigung der politischen Vorgaben der SED innerhalb des Dienstbetriebes kontrollierte. Befehle und Anordnungen der Kommandeure und Leiter erlangten erst mit der Zustimmung der PK-Leiter ihre Gültigkeit. Dem PK-Apparat oblag darüber hinaus auch die Anleitung der SED-Parteiorganisationen ${ }^{4}$.

Vor allem wegen des teilweise katastrophalen inneren Zustandes der VP-Bereitschaften und der mangelhaften Rekrutierungspolitik ${ }^{5}$ bestand allgemein eine schlechte Disziplin unter den Bereitschaftsangehörigen. Zudem gab es viele Desertionen. Dem versuchte die SED-Führung im Zuge des beginnenden Stalinisierungsprozesses ab Anfang 1949 durch eine breit angelegte Entlassungs- und Versetzungswelle zu begegnen'.

Vor dem Hintergrund der Gründung der NATO (North Atlantic Treaty Organization) und der sich abzeichnenden Teilung Deutschlands fiel in der ersten Jahreshälfte 1949 die Entscheidung, die kasernierten Polizeiverbände zu militärischen Kaderformationen auszubauen. Zunächst wurde im Juli die Grenzpolizei aus der HA GP/B herausgelöst. Auf Beschluß des DVdI-Präsidiums wurde die HA GP/B anschließend als selbständiger Dienstbereich innerhalb der DVdI in Verwaltung für Schulung (VfS) umbenannt. Leiter der VfS als zentralem Führungsorgan war der Vizepräsident der DVdI und spätere Minister für Staatssicherheit, Wilhelm Zaisser ${ }^{7}$. Der VfS kam vorrangig die Aufgabe zu, ein militärisches Offiziers- und Unteroffizierskorps heranzubilden, das den Kaderstamm künftiger Streitkräfte bilden sollte ${ }^{8}$.

Parallel dazu wurden die Bereitschaften der VfS gemäß sowjetischer Vorgaben umfassend umstrukturiert. Einerseits verringerte man die Zahl der Bereitschaften auf 35; andererseits aber wurde die Sollstärke je Bereitschaft auf rund 750 bis 1100 Mann erhöht. Zudem stieg die Zahl der VP-Schulen auf 11. Weiterhin erhielten die Schulen und Bereitschaften jetzt jeweils ein spezifisches Ausbildungsprofil und unterschieden sich entsprechend der verschiedenen Truppengattungen ${ }^{9}$. Im Herbst 1949 besaß die VfS eine Gesamtstärke von rund 35300 Mann. Mit den militärisch ausgebildeten und bewaffneten Polizeiverbänden der VfS verfügte die SED schon vor Gründung der DDR über ein Instrument der Machtsicherung im Innern ${ }^{10}$.

Die VfS war letztlich aber nur ein Zwischenschritt auf dem Weg zur Bildung von Organisationsstrukturen mit eindeutig militärischem Charakter, die die SED nach Gründung der DDR am 7. Oktober 1949 zunehmend forcierte. Nach der Bildung

4 Vgl. ebenda, S. $186 \mathrm{f}$.

5 Viele der Bereitschaftsangehörigen waren von sowjetischer Seite vor die Alternative gestellt worden, weiter in Kriegsgefangenschaft zu bleiben oder sich freiwillig für den Dienst zu verpflichten. Zudem waren viele der Geworbenen offenbar nicht bereit, in Verbänden zu dienen, die im Konfliktfall auch gegen westdeutsche Bürger eingesetzt werden sollten. Vgl. Diedrich/Wenzke, Die getarnte Armee, S. 28, 33 f.

$6 \mathrm{Vgl}$. ebenda, S. $33 \mathrm{f}$.

7 Auch alle übrigen Führungspositionen der VfS besetzte man mit SED-Kadern.

${ }^{8} \mathrm{Vgl}$. Eisert, Zu den Anfängen der Sicherheits- und Militärpolitik, S. $184 \mathrm{f}$.

924 VP-Bereitschaften gehörten zur Infanterie, acht zur Artillerie und drei zur Panzertruppe, dazu kamen fünf Infanterie-, drei Artillerieschulen und eine Panzer-, eine Polit- sowie eine Nachrichten- und Pionierschule.

10 Vgl. Eisert, Zu den Anfängen der Sicherheits- und Militärpolitik, S. 184 f. 
des Ministeriums des Innern (MdI), in das die DVdI integriert wurde, erfolgte die Umbenennung der VfS in Hauptverwaltung für Ausbildung (HVA), der als Führungsorgan nunmehr alle VP-Bereitschaften und Ausbildungseinrichtungen unterstanden. Der Leiter der HVA war dem Minister des Innern direkt unterstellt. Die Leitung der Verwaltung lag bis Februar 1950 bei Zaisser, dann zwei Monate lang bei Heinz-Bernhard Zorn und im Anschluß bei Heinz Hoffmann, der später, von 1960 bis 1985, Minister für Nationale Verteidigung war. Die HVA gliederte sich in den Stab, die Hauptabteilung Polit-Kultur, die Intendantur und die Abteilungen Personal, Allgemeines sowie Ausbildung und Inspektion. Der Ausbildung und Schulung in der HVA lag eine rein militärische Ausrichtung zugrunde. Im April 1950 erhielt sie von der UdSSR erstmals einige Panzer sowie schwere Geschütze und Granatwerfer. In den folgenden Monaten fand der Aufbau der HVA-Strukturen seinen vorläufigen Abschluß11.

Unter dem Eindruck des Korea-Krieges begann ab Anfang des Ausbildungsjahres 1950/1951 eine grundlegende Reorganisation der HVA. Die VP-Bereitschaften wurden auf 24 reduziert und nach dem Vorbild der Regimentsstruktur in den sowjetischen Landstreitkräften durchgehend einheitlich gegliedert. Dies sollte ein $\mathrm{Zu}$ sammenwirken der verschiedenen Waffengattungen nach militärischen Einsatzgrundsätzen ermöglichen ${ }^{12}$. Es bestanden jedoch weiterhin schwerwiegende Mängel in der fachlichen Entwicklung vieler VP-Angehöriger sowie in der Ausbildungsplanung und Dienstdurchführung insbesondere der Offiziere. Daraus resultierten vor allem eine schlechte innere Ordnung und eine anhaltend hohe Desertionsrate: 1950 gab es 600 Fahnenflüchtige, 1951 waren es 395. Die SED-Führung schätzte den Stand der politischen Zuverlässigkeit der HVA noch immer als unbefriedigend ein. Aus diesem Grund und wegen allgemein fehlender Eignung wurden 1950 rund 10000 VP-Angehörige vorzeitig aus dem Dienst entlassen, 1951 waren es nochmals 400013 .

Darüber hinaus hatte die SED insbesondere nach dem III. Parteitag im Juli 1950 damit begonnen, in der HVA die politisch-ideologische Erziehung systematisch zu verstärken. Die Partei strebte die Bildung eines dezidiert politischen Führerkorps an; vor allem von den Offizieren forderte sie ein klares Bekenntnis zur SED und die Wahrnehmung ihres politisch-ideologischen Erziehungsauftrages. Auch galt es den Anteil der Parteimitglieder in der HVA beständig zu erhöhen. 1951 gehörten von den knapp 52000 VP-Angehörigen insgesamt 31,5 Prozent der SED an; bei den Offizieren lag der Anteil sogar bei fast 60,0 Prozent ${ }^{14}$.

Nach Ablehnung der „Stalin-Note“ durch die Westmächte am 25. März 1952 und infolge der bevorstehenden Unterzeichnung des „Deutschland-Vertrages“ und des Vertrages über die Europäische Verteidigungsgemeinschaft (EVG) zeichnete sich für die UdSSR deutlich $a b$, daß die Westintegration der Bundesrepublik nicht mehr zu verhindern sein würde. Nachdem die gesamtdeutsche Option entfallen war,

11 Vgl. Diedrich/Wenzke, Die getarnte Armee, S. $40 \mathrm{ff}$.

12 Im Juni 1950 begann mit dem Aufbau der Hauptverwaltung Seepolizei (HVS) zudem die Schaffung einer DDR-Marine. Sie erreichte Ende des Jahres einen Personalbestand von insgesamt 22.80 Mann. Bis Anfang 1952 wurde die Aufstellungsphase der HVS im wesentlichen abgeschlossen. Vgl. Ehlert, Die Hauptverwaltung für Ausbildung, S. $273 \mathrm{ff}$.

13 Vgl. Ehlert, Die Hauptverwaltung für Ausbildung, S. 266 ff.

14 Wenzke, Auf dem Wege zur Kaderarmee, S. 242 ff. 
sollte die DDR nun nicht zuletzt auch militärpolitisch dauerhaft in das sozialistische Lager eingebunden werden. Anfang April 1952 wies die sowjetische Führung die SED daher an, in der DDR eine reguläre Armee aufzustellen. Demgemäß befahl der neue Minister des Innern Willi Stoph mit Wirkung vom 1. Juli 1952 die Bildung der KVP aus den Einheiten der HVA. Zugleich wurde die HVS in Volkspolizei-See (VP-See) umgewandelt und mit dem Aufbau der Volkspolizei-Luft (VP-Luft) begonnen. Offiziell war die KVP-Gründung ein Ergebnis der 2. Parteikonferenz der SED vom 9. bis 12. Juli 1952, auf der nicht nur die planmäßige "Errichtung der Grundlagen des Sozialismus" in der DDR, sondern auch die Schaffung "nationaler Streitkräfte" verkündet wurde ${ }^{15}$.

Unter dem Deckmantel der KVP begann der Aufbau kampffähiger militärischer Verbände. Der Minister des Innern selbst übernahm die direkte Führung der KVP. Sein 1. Stellvertreter und Chef des Stabes wurde Vincenz Müller. Dem Minister unterstand ferner als Stellvertreter für Politische Arbeit Rudolf Dölling, als Stellvertreter für Ausbildung Heinz Hoffmann, als Stellvertreter für Wirtschaftsfragen Bernd Weinberger sowie als Stellvertreter die Chefs der VP-See Waldemar Verner und der VP-Luft Heinz Keßler, ferner ab 1953 als Stellvertreter für Bauwesen Wilhelm Mayer ${ }^{16}$. Im Januar 1953 konstituierte sich als zusätzliches Beratungsgremium das Kollegium des MdI, dem Ulbricht als Vertreter des Politbüros angehörte. Parallel dazu wurde ab Juni 1952 mit der Territorialverwaltung Pasewalk nach sowjetischem Vorbild das erste Armeekorps der KVP aufgestellt. Der Territorialverwaltung, die über alle Waffengattungen verfügte, unterstanden zwei Infanteriedivisionen (Bereitschaften) und eine mechanisierte Division sowie weitere selbständige Kommandos und Abteilungen (Regimenter und Bataillone). Jede Division war ihrerseits in drei Regimenter untergliedert. Im Herbst begann die kadrierte Aufstellung der Territorialverwaltungen Dresden, Leipzig sowie Dessau, später Schwerin. Ab Mai 1952 wurde die HVA/KVP zudem in größerem Umfang mit sowjetischer Waffentechnik aus dem Zweiten Weltkrieg beliefert, darunter auch mittlere und schwere Kampfpanzer. Am 27. August 1952 befahl Stoph die Einführung militärischer Dienstgrade in der KVP, die im Dezember einschließlich der Luft- und Seeeinheiten eine Gesamtstärke von rund 90000 Mann erreichte ${ }^{17}$.

Im Rahmen des Ausbaus der militärischen Strukturen verstärkte die SED auch den Parteiapparat in der KVP. Zunächst wurde im Ministerium des Innern als politisches Führungsorgan eine Politische Verwaltung geschaffen. Dieser unterstanden die Politorgane aller Einheiten, die bis hinunter auf Kompanieebene existierten. Zur Überwachung ihrer Entscheidungen war jedem Kommandeur und Dienststellenleiter weiterhin ein Politoffizier zur Seite gestellt, der nunmehr als „Polit-Stellvertreter" bezeichnet wurde. Nachdem das Prinzip der doppelten Führung Ende 1952 aufgegeben und die sogenannte „Einzelleitung“ eingeführt worden war, erfolgte die Kontrolle durch die Politstellvertreter vor allem indirekt, um die Autorität der Kommandeure zu stärken und die Effizienz der Entscheidungsprozesse zu verbes-

$15 \mathrm{Vgl}$. Diedrich/Wenzke, Die getarnte Armee, S. $73 \mathrm{ff}$.

16 Als Fachreferate waren dem Minister zudem relativ selbständige Verwaltungen unter anderem für Panzer, Pionierwesen, Artillerieversorgung, Kfz, Bewaffnung, Finanzen und Kader unterstellt. Vgl. Diedrich/Wenzke, Die getarnte Armee, S. 106.

17 Vgl. Diedrich, Die Kasernierte Volkspolizei, S. $340 \mathrm{ff}$. 
$\operatorname{sern}^{18}$. Die Arbeit der Politorgane und Parteiorganisationen in der KVP erfolgte auf der Basis des „Statuts der Organe für Politarbeit in den nationalen Streitkräften der DDR" 19 . Ende 1952 gab es in den drei Teilen der KVP insgesamt über 3800 Politoffiziere 20 .

Der Volksaufstand am 17. Juni 1953 in der DDR, dessen unmittelbarer Auslöser die Normenerhöhung in der Industrie gewesen war, bedeutete den ersten ernsthaften Test für die Einsatzbereitschaft der KVP. Vor allem wegen des mangelnden Vertrauens der sowjetischen Besatzungsmacht in die politische Zuverlässigkeit kam die KVP während der Unruhen erst spät zum Einsatz, für den sie weder ausreichend ausgebildet noch ausgerüstet war. Am 17. Juni selbst wurden nur 8160 Mann eingesetzt. Die Einheiten hatten vor allem Räum- und Sicherungsaufgaben zu erfüllen, wurden in der Regel aber hinter den sowjetischen Truppen eingesetzt. Insgesamt zeigten sie sich nicht dazu in der Lage, die an sie gestellten Aufgaben zu erfüllen. Erst am folgenden Tag übernahm die KVP mit eindeutigem Schießbefehl hauptverantwortlich die Aufgabe, ein erneutes Aufflammen der Streiks und Demonstrationen zu verhindern. Letztlich bewies die KVP zwar, daß sie als Machtinstrument der SED verfügbar war. Ihr Einsatz während des Volksaufstandes machte aber deutlich, daß sie nach wie vor nicht über die militärische Einsatzbereitschaft und Führungsfähigkeit verfügte, um in einer derartigen Lage selbständig zu operieren. Obwohl sich die überwiegende Mehrheit der KVP-Angehörigen, die unmittelbar beim Aufstand eingesetzt wurden, als politisch zuverlässig erwies, kam es auch zu Desertionen 21 . Befehlsverweigerungen in der KVP und der VP-See wurden offiziell in nur rund hundert Fällen bekannt. Im Anschluß an den Volksaufstand gab es jedoch spürbar mehr Entlassungsgesuche, zudem stieg die Zahl der Desertionen stark an ${ }^{22}$. Diese Fahnenfluchten waren in einem hohem Maß politisch motiviert und verdeutlichten nicht zuletzt den Gewissenskonflikt, in dem sich viele KVP-Angehörige nach dem Einsatz gegen die eigene Bevölkerung befanden. In der Situation entließ die SED-Führung im Rahmen der durch die wirtschaftliche Krise erzwungenen Reduzierung der Gesamtstärke der KVP auf 99784 Mann auch politisch vermeintlich illoyales Personal; davon waren fast 25000 KVP-Angehörige betroffen ${ }^{23}$.

Darüber hinaus vollzog die SED eine Reorganisation des MdI, in dessen Folge Hoffmann am 1. August 1953 Stellvertreter des Ministers und Chef der KVP wurde. Zudem schuf man im September ein Kollegium der KVP, dem die wichtigsten Vertreter der KVP-Führung angehörten. Im Zuge der Verkleinerung der KVP

18 Vgl. S. $132 \mathrm{ff}$.

19 Statut der Organe für Politarbeit in den nationalen Streitkräften der DDR, 1952, BA-MA, DVH 3/3408, Bl. 3 ff. Das Statut wurde 1954 modifiziert.

20 Vgl. Diedrich/Wenzke, Die getarnte Armee, S. 211.

21 Diesbezüglich äußerte ein Oberst der KVP: „Wer am 17. Juni mitgemacht hat ... hier in Berlin, der weiß, daß unsere Volkspolizisten in den ersten Tagen eine wirkliche Begeisterung zeigten - aber wir wissen auch, ... wieviele Desertionen es gab, gerade bei diesen Einheiten, die hier in Berlin ihre Aufgabe erfüllten. "Protokoll der Parteidelegiertenkonferenz der KVP zur Vorbereitung des IV. Parteitages der SED, 8.-10. März 1954, BA-MA, DVH 3/3416, Bl. 225.

22 Im September 1953 erhöhte sich die Zahl der Fahnenfluchten gegenüber Januar um 198 Prozent. Im gesamten Jahr desertierten insgesamt 85 KVP.-Offiziere. Protokoll Nr. 8/55 der Sitzung des Kollegiums der KVP, 19. Dezember 1955, BA-MA, DVH 3/2055, Bl. 41.

$23 \mathrm{Vgl}$. Diedrich/Wenzke, Die getarnte Armee, S. $319 \mathrm{ff}$. 
blieben nur noch die Armeekorps Nord und Süd mit jeweils rund 30000 Mann bestehen, die in drei Divisionen gegliedert waren. Ferner gab es die mechanisierte Bereitschaft Potsdam und 21 Schulen der verschiedenen Waffengattungen. Diese Grundstruktur änderte sich bis zur NVA-Gründung nur noch geringfügig. Insgesamt begann im Ausbildungsjahr 1953/1954 eine Phase der Konsolidierung für die KVP. Personell, organisatorisch und hinsichtlich des Ausbildungsstandes sollten die Grundlagen für den Ausbau der KVP zu regulären Streitkräften geschaffen wer$\operatorname{den}^{24}$.

Nachdem die Ostblock-Staaten im Dezember 1954 beschlossen hatten, im Falle des Inkrafttretens der „Pariser Verträge“ vom 23. Oktober des Jahres ein eigenes Militärbündnis zu gründen, leitete die SED-Führung nun endgültig die Umwandlung der KVP als getarnte Militärorganisation in eine schlagkräftige Kaderarmee ein. Diese wurde nach der Aufnahme der Bundesrepublik in die NATO am 5. Mai 1955 weiter intensiviert. Nach Einbeziehung der DDR in den am 14. Mai 1955 gegründeten Warschauer Pakt war es das Ziel der SED, die militärische Leistungsfähigkeit der zukünftigen Streitkräfte, die von vornherein als Koalitionsarmee konzipiert wurden, möglichst schnell an die der übrigen Bündnisarmeen anzugleichen. Durch die Ergänzungen der Verfassung von 1949, die die Volkskammer am 26. September 1955 beschloß, erhielt die DDR formal das Recht zur Landesverteidigung und damit auch zur Aufstellung einer regulären Armee ${ }^{25}$.

24 Vgl. ebenda, S. $363 \mathrm{ff}$.

25 Diedrich, Die Kasernierte Volkspolizei, S. 357. 